\title{
Carbon Migration During Methane Injection Experiments Under ITER Divertor-Relevant Conditions
}

\author{
K. Bystrov ${ }^{\mathrm{a}^{*}}$, L.B. van der Vegt ${ }^{\mathrm{a}}$, G.A. van Swaaij ${ }^{\mathrm{a}}$, T. Zaharia ${ }^{\mathrm{a}, \mathrm{b}}$, Y. Kuang ${ }^{\mathrm{c}}$, \\ W.J. Goedheer ${ }^{a}$ and G. De Temmerman ${ }^{a}$. \\ ${ }^{a}$ FOM Institute DIFFER - Dutch Institute for Fundamental Energy Research, Association \\ EURATOM-FOM, Partner in the Trilateral Euregio Cluster, P.O. Box 1207, 3430 BE \\ Nieuwegein, The Netherlands. \\ ${ }^{b}$ Eindhoven University of Technology, Department of Applied Physics, Plasma and Material \\ Processing, P.O. Box 513, 5600 MB Eindhoven, The Netherlands. \\ ${ }^{c}$ Utrecht University, Faculty of Science, Department of Physics and Astronomy, Nanophotonics - \\ Physics of Devices, P.O. Box 80 000, 3508 TA Utrecht, The Netherlands.
}

\begin{abstract}
Methane was injected into a high density low temperature hydrogen plasma beam $\left(n_{e} \leq 10^{21} \mathrm{~m}^{-3}, T_{e} \sim 1 \mathrm{eV}\right)$ in Pilot-PSI to study carbon transport and provide a benchmark case for modeling. Gas injection was performed through molybdenum samples that acted as collectors of redeposits. The $\mathrm{CH}$ A-X band emission plumes are localized near the injection hole with a typical width of $\sim 3 \mathrm{~mm}$ and a decay length of $\sim 1.5 \mathrm{~mm}$. The geometry of the emission profile is not influenced by the electron temperature. The redeposition efficiency and codeposit structure depend on the surface temperature. Surface temperatures below $500 \mathrm{~K}$ promote growth of a-C:H films across the surface and lead to redeposition of up to $38 \%$ of injected carbon. Above $800 \mathrm{~K}$ the redeposition efficiency decreases strongly and carbon microparticles are found near the injection hole.
\end{abstract}

PSI-20 keywords: Amorphous films, Carbon impurities, Erosion \& Deposition, Hydrocarbons, Surface analysis.

PACS: $52.25 \mathrm{Xz}, 52.40 . \mathrm{Hf}, 52.70 . \mathrm{Kz}$

Corresponding Author Address: FOM Institute DIFFER - Dutch Institute for Fundamental Energy Research, P.O. Box 1207, 3430 BE, Nieuwegein, The Netherlands

Corresponding Author e-mail: k.bystrov@differ.nl

Presenting Author: Kirill Bystrov

Presenting Author e-mail: k.bystrov@differ.nl 


\section{Introduction}

During operation of fusion devices plasma-facing materials can be eroded, transported to a new location and redeposited [1]. Material migration is important because of the limitations it sets on components lifetime and impurity concentration in the plasma. If carbon is used in the divertor its codeposition with hydrogen isotopes in remote areas becomes arguably the biggest concern [2]. It becomes extremely challenging to prevent tritium accumulation in areas, that are difficult to access.

Since a significant fraction of carbon in fusion devices is eroded in the form of hydrocarbons, local hydrocarbon injection became a useful tool for studying carbon migration both in tokamaks [3] and linear devices [4, 5]. Despite numerous studies, the understanding of carbon transport is still limited by unknowns such as sticking coefficients of different species or re-erosion rates of codeposited species [6]. We have performed a series of methane injection experiments in the linear plasma generator PilotPSI to address the questions of hydrocarbons transport in the plasma and the spatial extent of carbon redeposition. The unique feature of Pilot-PSI is the ability to create a high density low temperature $\left(n_{e} \leq 10^{21} \mathrm{~m}^{-3}, T_{e} \sim 1 \mathrm{eV}\right)$ plasma beam for up to $160 \mathrm{~s}$, entering the ITER divertor-relevant regime. The experimental results are compared to simulations with the 3D Monte Carlo impurity transport and PSI code ERO [7, 8].

\section{Experimental}

The Pilot-PSI linear plasma generator is described in detail in [9]. Magnetic field strength of $0.4 \mathrm{~T}$ was used throughout this work. Unless stated otherwise, hydrogen was used as the process gas. 
Plasma electron density $n_{e}$ and temperature $T_{e}$ are measured by Thomson scattering at a distance of $\sim 25 \mathrm{~mm}$ upstream from the plasma facing surface [10]. Both $n_{e}$ and $T_{e}$ peak in the middle of the plasma beam and decrease with distance from the axis. Throughout this article we refer to the peak values of density and temperature. Typical full width at half maximum (FWHM) of the plasma beam is $\sim 12 \mathrm{~mm}$.

Methane was injected into the center of the plasma column through a single hole in polished molybdenum samples. The diameter of the injection hole was $0.6 \mathrm{~mm}$. The injection rate was set at $3 \mathrm{sccm}$ (standard cubic centimeter per minute; $1 \mathrm{sccm}=4.4 \times 10^{17}$ $\mathrm{CH}_{4} / \mathrm{s}$ ). The samples had a diameter of $25 \mathrm{~mm}$. We used the samples as collectors for the deposits. Importantly, injection of methane had no influence on the measured profiles of $n_{e}$ and $T_{e}$.

Optical emission spectroscopy was used to quantify the penetration of the injected methane into the main plasma. We monitored the molecular $\mathrm{CH}$ A-X band emission (Gerö band around $431 \mathrm{~nm}$ ) using an absolutely calibrated CCD camera equipped with an interference filter (transmission peak at $430.7 \mathrm{~nm}$, FWHM of $2.7 \mathrm{~nm}$ ). A correction factor of 2.8 was used to determine the intensity of the full $\mathrm{CH}$ A-X band from the measurements in the reduced wavelength range [11]. The camera was positioned to have a tangential view of the sample and was focused on the location of injection. Detailed description of the mechanisms that lead to the creation of the excited $\mathrm{CH}$ molecules in Pilot-PSI plasmas can be found in [12].

A fast infrared camera (SC7500-MB, FLIR) was used to monitor the surface temperature of the samples during exposure. After the exposure, analyses of the samples 
included mass gain measurements, scanning electron microscopy (SEM), spectroscopic ellipsometry and visible Raman spectroscopy.

\section{Results}

Spectroscopic observations of the CH A-X band are summarized in Figure 1. The emission profiles are plotted along and across the magnetic field axis. The emission plumes were recorded for three sets of plasma parameters, as indicated in the figure. The $\mathrm{CH}$ emission is localized in the immediate vicinity of the injection point. The $e$-folding length of the profile in the axial direction is equal to $1.3-1.5 \mathrm{~mm}$. In the direction across the axis the emission has a Gaussian profile with FWHM in the range 2.5-3.0 mm which is significantly smaller than the diameter of the plasma beam. The shape of the plume is independent of the $T_{e}$ in the range from $0.3 \mathrm{eV}$ to $2.2 \mathrm{eV}$. In the previous methane injection experiments [13] the hole for methane injection was almost two times larger, however the $\mathrm{CH}$ emission plumes were quite similar compared the ones obtained in this study. Additionally, we have varied the injection rate from $0.6 \mathrm{sccm}$ to $3 \mathrm{sccm}$ and noticed no significant effect on the plume dimensions.

Hydrocarbon transport in the plasma, erosion and redeposition are strongly interdependent parts of carbon migration. Understanding migration requires that its constituents are studied together. That was the reason for collecting redeposits using the same injection set-up. The samples were exposed at different surface temperatures, while the methane injection rate was kept the same. Mass gain measurements were used to determine the net amount of redeposited carbon. The results are summarized in Table 1, where $T_{\text {surf }}$ is the surface temperature as measured by the IR camera, $R$ is the fraction of 
injected carbon redeposited on the sample, $T_{e}$ is the electron temperature, $n_{e}$ is the electron density and $t$ is the exposure time. Since the FWHM of the plasma beam is smaller than the diameter of the samples, the temperature is non-uniform across the surface. This is why the temperature span is given in the table. Examples of the surface temperature profiles are given in Figure 2. The profiles feature sharp peaks (dashed lines in Figure 2) in the immediate vicinity of the injection holes. This effect could be due to overheating of the edges of the injection hole. At the same time, the injection hole can act as a small cavity trapping the radiation and acting close to a black body. In any case, this effect was very localized. Excluding the spike, the shape of the temperature profiles is consistent with the Gaussian shape of the plasma beam.

Note that under hydrogen plasma bombardment the total amount of redeposited carbon increases at lower surface temperatures. Yet the largest fraction of carbon was redeposited in a single experiment with pure argon plasma $\left(n_{e}=5.5 \times 10^{20} \mathrm{~m}^{-3}, T_{e}=1.3\right.$ $\mathrm{eV})$. The energy of argon ions was insufficient to cause sputtering of carbon. This shows how important chemical re-erosion is in establishing the balance between net deposition and erosion.

The morphology and localization of the deposits are also strongly influenced by the temperature. Below $500 \mathrm{~K}$ hydrogenated amorphous carbon (a-C:H) films are deposited over the entire exposed surface (Figure 2). The films are the thickest close to the injection point. In this region they are unstable in air and flakes peel away in a matter of hours. The rest of the sample is covered by a thinner film resilient to the air exposure. When the temperature is larger than $800 \mathrm{~K}$ no films are deposited on the surface. Instead, a narrow ( $\sim 5 \mathrm{~mm}$ in diameter) region around the injection hole gets covered by dense 
agglomerations of carbon microparticles (Figure 3). Previously, particles with similar size and fine-structure were found on fine-grain graphite samples under similar conditions, but without methane injection [14]. The influence of plasma conditions on the particle growth and studies of their internal structure are described in [15]. In the intermediate temperature range we observed the microparticles embedded into the amorphous film. Interestingly, samples 1 and 3, that were exposed under similar plasma conditions exhibit different redeposit morphologies. This illustrates the crucial role of the surface temperature.

A characteristic visible Raman spectrum of the microparticles is given in Figure 4. The spectrum is dominated by the so-called D-peak and G-peak, with the ratio of the peak intensities $I_{D} / I_{G} \sim 2$. The double-peaked spectrum resembles measurements of carbon layers from Tore Supra [16] and carbon dust samples from NSTX [17]. The authors of [16], who also performed X-ray diffraction studies, attributed such spectral shape to high porosity of the samples and concluded that they are highly disordered carbons consisting mainly of $\mathrm{sp}^{2}$ bonds. However, other forms of carbon have similar structure of the Raman spectrum [17]. More detailed measurements are needed for a definite conclusion concerning the structure of the microparticles in our experiments. It should be mentioned that despite their large size, those particles account for a small (< $10 \%)$ fraction of the injected carbon, and that their hydrogen content is very low.

Refractive index $n$ and the film thickness have been measured by spectroscopic ellipsometry at several locations along the radius of the samples. The thickness of the films decreases towards the edge of the samples (Figure 5). This is not surprising, since the source of carbon was located in the center of the samples. Comparison of the 
measured refractive indexes with values documented in the literature [18] allowed us to estimate a film density of about 0.8 g.cm ${ }^{-3}$ and a hydrogen content of 55 at. \%. Of course, this is a rough estimation since the film properties change with the distance from the center. In fact, since refractive indexes are decreasing towards the sample edge (Figure 5), the hydrogen content is expected to increase along the sample radius which could be explained by the lower temperatures on the periphery.

After $100 \mathrm{~s}$ of growth the film thicknesses reach several hundred nanometers on the edge of the samples. The thicknesses of several micrometers are measured approximately $5 \mathrm{~mm}$ away from the injection hole. Ellipsometry measurements closer to the center were not possible due to flaking of the films, although the flaking indicates even larger thicknesses. Growth rates of the order of hundreds of nanometers per second are predicted by ERO [8]. This is compatible with the observed thicknesses of the films. Growth rates of the same order of magnitude are obtained from mass gain measurements assuming uniform growth across the sample surface (horizontal lines in Figure 5).

\section{Discussion}

Carbon redeposition was observed during all methane injection experiments in Pilot-PSI. However, both overall redeposition efficiency and radial profiles of redeposition vary significantly with surface temperature while they appear insensitive to the plasma parameters. The two extreme scenarios in terms of morphology are a-C:H films covering the full surface of the sample at low temperatures and very localized dustlike deposits at high temperatures. 
In these experiments, growth rate of deposits at any given location is determined by the balance between redeposition and erosion of carbon. In the case of argon plasma, when the deposited carbon could not be re-eroded, the redeposited fraction calculated with ERO was $65.0 \%$ and within the experimental error bar. Although the redeposition profile does not match exactly, simulation and experiment are in the same order of magnitude [8]. In the case of hydrogen plasma, ERO simulations were performed assuming constant, but varied from run to run, value for chemical erosion of carbon [8]. If an erosion yield of $0.2 \%$ is assumed, ERO results are in good agreement with growth rates on the edge of the samples. However, the total amount of redeposited carbon is in this case overestimated. For a better match with the mass gain measurements the yield of $5.0 \%$ has to be assumed, but this means suppression of any film growth on the outside of the sample. A plausible explanation could be a variation of erosion yield across the surface. Indeed, the erosion yield is known to be a complicated function of surface temperature and ion energy [19], particle flux [20] and properties of the films [21], that is not yet well understood in the high plasma flux regime. So, a gradient of the erosion yield could be caused by the narrow width of the plasma beam compared to the sample size and associated gradients in plasma parameters and surface temperature. The application of the Roth model of chemical sputtering $[19,20]$ to these experiments is not straightforward. As mentioned earlier, carbon films grow in the low surface temperature regions under bombardment by low energy ions, albeit the plasma flux is high. The local balance in these regions is shifted from erosion to deposition, yet the gross erosion is not zero. Indeed, earlier experiments [22] demonstrated that graphite is eroded under such conditions, but the Roth model predicts otherwise. Our experimental conditions are 
outside the applicability region of the model and quantification of the chemical erosion yield profile across the sample surface in Pilot-PSI is the subject of future work.

Flaking of thick films close to the injection point did not allow us to establish the growth rates there. Thus, a comparison of characteristic widths of the plasma, emission plumes and deposition profiles was not possible. Lower injection rates and shorter exposure times might help to overcome this hurdle.

\section{Conclusions}

Injection of methane through the sample into the center of hydrogen plasma beam in Pilot-PSI results in localized CH A-X emission plumes. The emission intensity decays rapidly both along and across the magnetic field axis. The plume shape is unaffected by the electron temperature. The amount of carbon redeposited on the sample, its distribution over the surface and morphology of the deposits depends strongly on the surface temperature. For $T_{\text {surf }}<500 \mathrm{~K}$ a-C:H film grows across the entire surface, its thickness being maximal close to the injection point. If $T_{\text {surf }} \geq 800 \mathrm{~K}$ no visible film growth is observed, instead deposition occurs only in the center of the sample. The deposits in this case are in the form of carbon microparticle agglomerates. There also exists an intermediate phase when particles are embedded in the film. A balance between

local deposition and erosion is the key to explain the observed changes in the location of the redeposits. For the same plasma parameters, surface temperature variations change local erosion yield significantly, thus shifting the balance in one way or the other.

\section{Acknowledgement}


The authors would like to thank Richard Al, Marc van de Pol, Hennie van der Meiden and the rest of the Pilot-PSI team for their support in preparation and execution of the experiments. This work was supported by the European Communities under the contract of Association between EURATOM/FOM and carried out within the framework of the European Fusion Programme with financial support from NWO and the NWO Grant No. RFBR 047.018.002. The views and opinions expressed herein do not necessarily reflect those of the European Commission. 


\section{References}

[1] R. Pitts, et al., Plasma Phys Controlled Fusion 47 (2005) B303-B305

[2] R.P. Doerner, et al., Nucl Fusion 49 (2009) 035002

[3] S. Brezinsek, et al., J Nucl Mater 363-365 (2007) 1119-1128

[4] D.G. Whyte, et al., Nucl Fusion 41 (2001) 47-62

[5] D. Naujoks, et al., Phys Scr T111 (2004) 80-85

[6] A. Kirschner et al., J Nucl Mater 415 (2011) S239-S245

[7] A. Kirschner, et al., Nucl Fusion 40 (2000) 989-1002

[8] G.A. van Swaaij, et al., these proceedings

[9] G.J. van Rooij, et al., Appl Phys Lett 90 (2007) 121501

[10] H.J. van der Meiden, et al., Rev Sci Instrum 79 (2008) 013505

[11] S. Brezinsek, et al., Phys Scr 111 (2004) 42-48

[12] G.A. van Swaaij, et al., Plasma Phys Controlled Fusion 54 (2012) 095013

[13] J. Westerhout, et al., Nucl Fus 50 (2010) 095003

[14] K. Bystrov, et al., J Nucl Mater 415 (2011) S149-S152

[15] K. Bystrov, et al., J Vac Sci Technol A 31 (2013) 011303

[16] P. Roubin, et al., J Nucl Mater 337-339 (2005) 990-994

[17] Y. Raitses, et al., J Nucl Mater 375 (2008) 365-369

[18] W. Jacob, Thin Solid Films 326 (1998) 1-42

[19] J. Roth, J Nucl Mater 266-269 (1999) 51-57

[20] J. Roth, et al., J Nucl Mater 337-339 (2005) 970-974

[21] A. Litnovsky, et al., Fusion Eng Des 86 (2011) 1780-1783 
[22] J. Westerhout, Carbon chemical erosion in high flux and low temperature hydrogen plasma, Eindhoven University of Technology Ph.D. Thesis, July 2010. 


\section{Figure captions}

Table 1. Exposure conditions, redeposited fractions $(R)$ and morphologies of the carbon deposits for various samples. Column $T_{\text {surf }}$ describes the variation of the temperature from the edge of the sample towards the center.

Fig 1. Axial (top) and radial (bottom) profiles of the $\mathrm{CH}$ A-X emission measured by optical emission spectroscopy. The dashed line marks the position of the sample obtained from the background images taken before the experiment. Small misalignment or defocusing of the camera cause uncertainty in determining the position of the injection nozzle, which would explain the signal in the $x<0$ region.

Fig. 2. (Top) Temperature profiles across the surface of the samples as measured by the infrared camera. Dotted lines indicate the region of uncertainty in the temperature measurement. (Bottom) Photographs of the samples with different types of deposits. Fig. 3. SEM images of the carbon microparticles near the injection hole.

Fig. 4. Visible Raman spectra of the carbon microparticle deposits near the injection hole. Fig. 5. Growth rates, film thicknesses (both - top) and refractive indexes (bottom) of the deposited films as a function of the distance from the center. Samples numbering is taken from Table 1. Horizontal lines in the upper figure - calculated growth rates from mass gain measurements assuming homogeneous growth across the surface. 


\begin{tabular}{ccccccccc}
$\begin{array}{c}\text { Sample } \\
\text { ID }\end{array}$ & $\mathbf{T}_{\text {surf }}, \mathbf{K}$ & $\mathbf{R}, \mathbf{\%}$ & $\begin{array}{c}\text { Morphology } \\
\text { Center }\end{array}$ & Edge & $\mathbf{T}_{\mathbf{e}}, \mathbf{e V}$ & $\begin{array}{c}\mathbf{n}_{\mathbf{e}}, \mathbf{m}^{-3} \\
\left(\times 10^{20}\right)\end{array}$ & $\begin{array}{c}\mathbf{t}, \\
\mathbf{s e c}\end{array}$ \\
1 & $1020-1300$ & n/a & MP & ND & 2.2 & 1.8 & 140 \\
\hline 2 & $825-975$ & $<10$ & MP & ND & 1.8 & 1.6 & 500 \\
\hline 3 & $415-695$ & $14 \pm 5$ & F+MP & F & 2.2 & 1.8 & 100 \\
\hline 4 & $350-425$ & $21 \pm 8$ & F & F & 0.3 & 1.6 & 100 \\
\hline 5 & $365-415$ & $38 \pm 8$ & F & F & 0.3 & 2.0 & 100 \\
\hline Argon & $\leq 525$ & $54 \pm 22$ & F+MP & F & 1.3 & 5.5 & 40
\end{tabular}




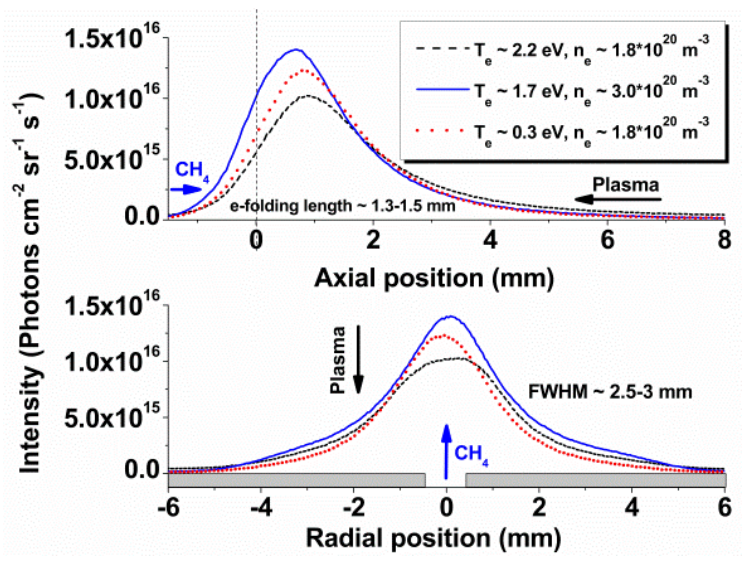

Fig 1. 

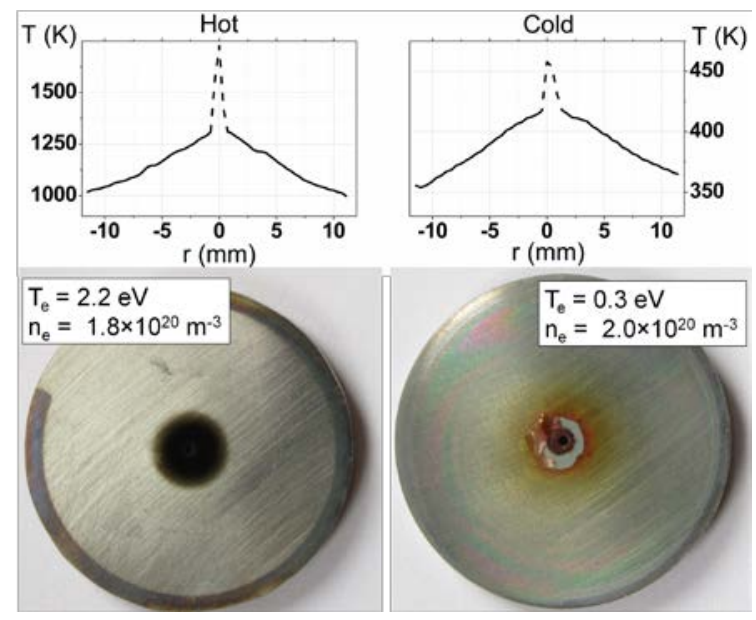

Fig. 2. 


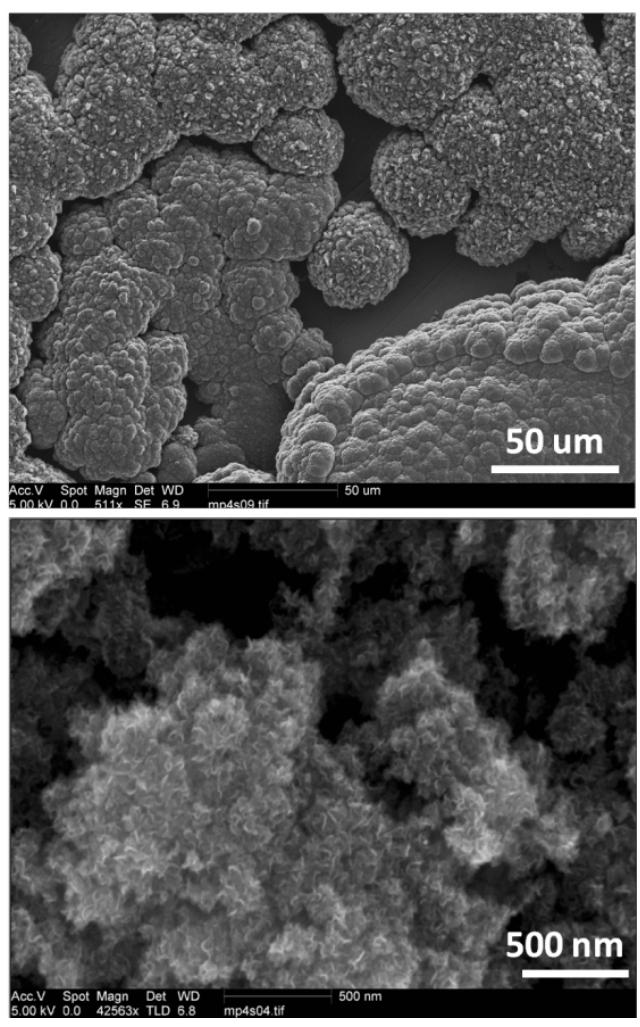

Fig. 3. 


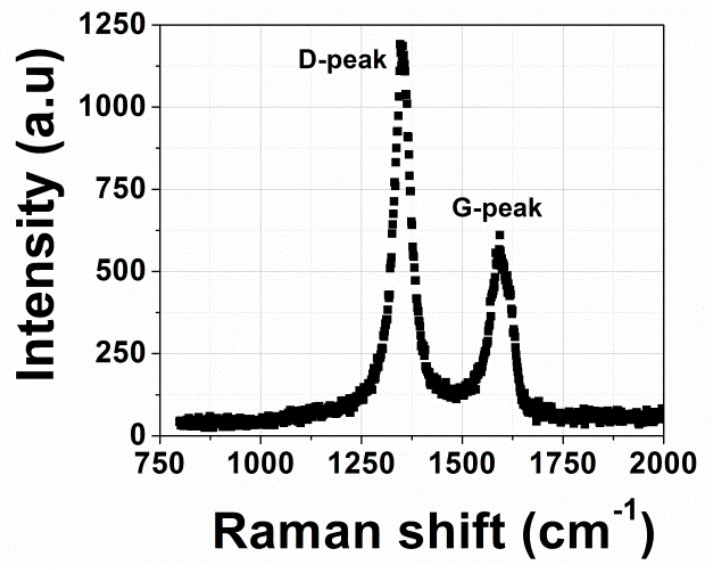

Fig. 4. 

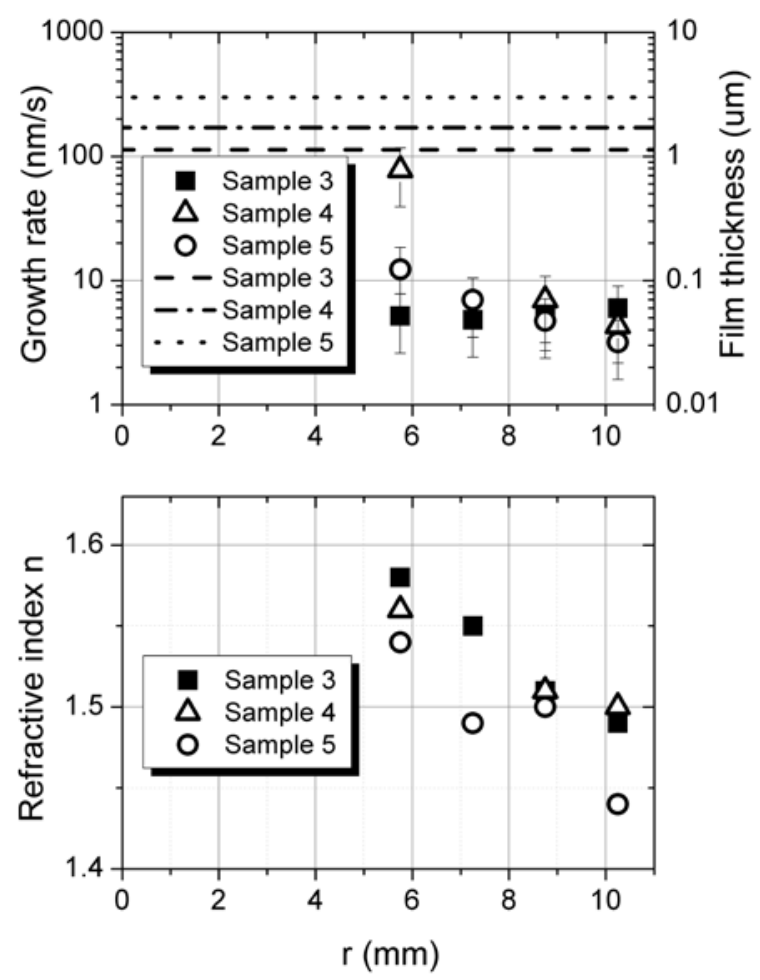

Fig 5. 